\title{
AUDITING OF THE COMPUTERISED INFORMATION SYSTEMS IN THE MILITARY ORGANIZATION
}

\author{
Valentin PÎRVUȚ \\ pirvut_v@yahoo.com \\ “NICOLAE BĂLCESCU” LAND FORCES ACADEMY, SIBIU, ROMANIA
}

\begin{abstract}
An internal public auditing mission is a complex process with multiple objectives and auditable objects, which offers a most complete management of the audited entity's identified risks and proposes solutions and recommendations for limiting their impact. In the informatics segment, the calculators, informatics systems (the operation systems, the informatics applications used in the entity, the accounting management systems of the data bases), the communication networks, the procedures and operations adjacent to the informatics systems, the policies in the system are subjected to control and evaluation. To this end, due to the importance the use of the calculator grants within the entities, in some countries there have been elaborated several normative acts regulating the use, control and auditing of the electronic devices used for processing the data. Thus, the most important regulations referring to the auditing within the computerized information systems (CIS - Computerized Information Systems) are the ISA (International Standards on Auditing) and IAPS (International Auditing Practice $\boldsymbol{S}$ tatement) standards elaborated by IFAC (International Federation of Accountants).
\end{abstract}

\section{KEYWORDS:}

Auditing, accounting management, control, devices, computerised information systems 


\section{Introduction}

In the specialized literature and practice, the aspects related to the risks and control of using the technology of information fall under the term IT Governance, the conduct and coordination of the technology of information in an organization respectively.

IT Governance practically represents the process of control and coordination of informational resources, such as: the devices of receival, processing, storage and automatic transmission of data (calculators, peripherals, networks, servers, routers, computerized information applications, etc.), the policies and procedures governing the computerized information processes, the users, the staff involved in developing and managing the informational system (IT analysts, programmers, managers, etc.), suppliers of informational resources and the auditors of the informational system.

IT Governance's view is that these resources must be used converging with the organization objectives and strategies, but with minimum risks.

The main objectives of IT Governance are:

$\checkmark$ establishing strategies so that the use of informational resources is in accordance with the organization objectives;

$\checkmark$ the most efficient use and with minimum risks of informational resources.

The factors which determine the pursuit and evaluation of control procedures in the computerised information business environments can be summarily grouped in:

$\checkmark$ cost of data failure;

$\checkmark$ cost of making wrong decisions;

$\checkmark$ cost of abuse in the computerised information system;

$\checkmark$ increased value of the calculus system, of the computerised information applications and of the specialized personnel;

$\checkmark$ increased cost of the errors resulting from calculators;

$\checkmark$ confidentiality protection;

$\checkmark$ control of the evolution of the way in which calculators are used.

Synthesising the above-mentioned aspects, it is imperative that the auditing of a computerised information system should be done as an act of certifying the correctness and security of the operations in this system.

Throughout time, the necessity of an activity of computerised information auditing has emerged as a result of the following considerations:

$\checkmark$ The financial and internal auditors realised that the computerised information system has an increasing impact on the objectives of their missions;

$\checkmark$ The managers of the entities acknowledged the calculator as being the key resource in the market competition, which results in the necessity of control and auditing of the processes where it is used;

$\checkmark$ The professional associations and organizations, as well as governments have recognized the necessity of controlling and auditing the computerised information systems.

The impact of computerised information systems on the entity is embodied, without being limited, in:

$\checkmark$ increase in the security of computerised information devices;

$\checkmark$ increase in the security of data integrity;

$\checkmark$ increase in the effective use of the computerised information system;

$\checkmark$ increase in the employment efficiency of the computerised information system;

$\checkmark$ increase in the efficiency and quality of the security procedures and policies;

$\checkmark$ increase in the quality of internal control:

a. of security;

b. increase in the quality of internal control.

2. Definition and Content of Computerised Information Systems Auditing

In the literature and practice of Romania, the terms computerised information systems auditing, informational systems auditing or IT auditing are to be found. The conceptual difference between these terms is given either by the content and level at which the auditing activity takes place or by the conceptual difference existing between the notions informational system and IT system.

Thus, the computerised information system auditing is conceptually the most comprising of them, covering its objectives, all the levels of the computerised information system, from the evaluation of design and use of the computerised information system to the evaluation of the security policies and procedures at the operational and strategic level. 
The computerised information system auditing, the IT auditing respectively, covers through its objectives only the computerised information system.

In a word, the computerised information system auditing conceptually contains the IT auditing of the computerised information system. Since, in most entities, the computerised information system covers almost all the informational system, the most frequently used terms will be the computerised information system auditing or the IT audit, while for the auditor the terms auditor of computerised information systems or IT auditor will be used.

The computerised information system auditing represents the activity of collecting and evaluating some evidence for determining whether the information system is secured, it maintains the integrity of the processed and stored data, it allows for the accomplishment of the enterprise strategic objectives and uses the informational resources efficiently.

As a rule, this activity has to be carried out by personnel that is trained and qualified in the field of control, security and management of computerised information systems. Professional acknowledgement of this qualification is provided by CISA (Certified Information $\boldsymbol{S} y$ stems $\boldsymbol{A}$ uditor) certificate issued by ISACA (Information Systems Audit and Control A ssociation).

Although there is a quite close methodological link between the financialaccounting auditing and the computerised information system auditing, the latter is based on knowledge belonging to at least four domains, such as: traditional auditing, computerised information systems for management, behaviour science (psychology) and computer science.

The computerised information auditing can be organized at the level of the entity, within the internal auditing function, as well as in the form of external auditing carried out by personnel outside the entity.

In a mission of a computerised information system, the most frequent operations are the verifications, evaluations and testing's of the informational devices, such as:

$\checkmark$ identification and evaluation of risks in the system; the system; $\checkmark$ verification and physical evaluation of the informational environment;

$\checkmark$ verification and evaluation of the computerised information system administration;

$\checkmark$ verification and evaluation of the computerised information applications;

$\checkmark$ verification and evaluation of the security of calculator networks;

$\checkmark$ verification and evaluation of retrieval plans and procedures in case of disasters and of activity continuation;

$\checkmark$ testing the data integrity.

\section{Knowledge and Skills of a} Computerised Information Systems Auditor

Taking into account the fact that the auditing of computerised information systems is a discipline which involves specialized knowledge in several fields, the profession of computerised information systems auditor is a complex and extremely dynamic one.

In international specialized practice, there are several recommendations both with regard to the knowledge necessary to an auditor of computerised information systems and to the training of such an auditor, such as:

$\checkmark$ ISACA guidelines (2004), in which 040.010 and 040.020 paragraphs refer to the knowledge and aptitudes of the auditor of computerised information systems, to his continuous professional training respectively;

$\checkmark$ IFAC (2003) standard in which ISA 401 refers to the aptitudes and competence of a financial auditor in the conditions of an auditing in a computerised information medium.

Synthesizing, the knowledge that an auditor of computerised information systems must have and acquire, the following can be enumerated:

$\checkmark$ knowledge in the field of financial audit;

$\checkmark$ knowledge in the field of management;

$\checkmark$ knowledge in the field of accounting;

$\checkmark$ knowledge in the financial field;

$\checkmark$ knowledge regarding the evaluation of risks;

$\checkmark$ knowledge regarding control;

$\checkmark$ knowledge regarding the hardware of computerised information systems;

$\checkmark$ knowledge regarding the systems of operation and the computerised information applications; 
knowledge

telecommunications;

$\checkmark$ knowledge regarding the security of computerised information systems;

$\checkmark$ knowledge regarding the analysis and design of computerised information systems;

$\checkmark$ knowledge regarding programming and the programming languages;

$\checkmark$ knowledge regarding the accounting management systems of the data bases;

$\checkmark$ knowledge regarding the techniques of automatic processing of data within the computerised information systems of accounting management;

$\checkmark$ basic knowledge of statistics;

$\checkmark$ knowledge regarding legislation.

This knowledge can be acquired either within the higher education institutions, through postgraduate specialization and professional courses, or through certifications. The auditor of computerised information systems must also attend continuous professional courses and specialized conferences. To this end, the auditor should adhere to specialized professional bodies and organisms.

In order to acquire the aptitudes of the auditor of computerised information systems, this must:

$\checkmark$ be a good member in an auditing team;

$\checkmark$ be a good manager of the auditing activities;

$\checkmark$ have a well-developed observation spirit;

$\checkmark$ be a good collaborator;

$\checkmark$ have communication abilities; be able to make objective decisions;

be a good IT analyst.

\section{Conclusions}

The methodological framework associated with a mission of internal public auditing aims at obtaining an understanding of the organization requirements, the identification of the existing controls, the evaluation of internal control conformity through the identification of risks and the recommendation of improving its performance. The principles of auditing are formed around these assumptions.

The verification of the data integrity is a preliminary stage of accomplishing the auditing mission because auditors have to make sure that the results in the final reports are based on complete, accurate and reliable data. The errors or even frauds carried out by means of the calculator are not few. The internal control in a computerised information system generally aims at a general control affecting the medium of computerised information activities and the control of the applications existent in the system.

From the point of view of the practice of auditing, there is no clear distinction between the two types of control since they actually complete each other and together they provide a complete and fair image of the computerised information system.

\section{REFERENCES}

http://www.ifac.org

http://www.isaca.org

http://www.theiia.org

Information Systems Audit and Control Association. (2004). CISA Review Manual, Rolling Meadows, Retrived from: http://www.isaca.org

International Federation of Accountants. (2003). International Standards on Auditing (ISA), Retrived from: http://www.ifac.org 